\title{
General position theorem and its applications
}

\author{
Vladislav Aseev
}

Kirill Kamalutdinov

Andrey Tetenov *

\section{Introduction}

Consider the following problem:

Let $K$ be the attractor of a system $\mathcal{S}=\left\{S_{1}, \ldots, S_{m}\right\}$ of contraction maps in $\mathbb{R}^{n}$, and let $\operatorname{dim}_{H} K<$ $n / 2$. Suppose that the intersection $S_{i}(K) \cap S_{j}(K)$ is nonempty for some $i, j$. Is it possible to change the maps $S_{k} \in \mathcal{S}$ slightly to maps $S_{k}^{\prime}$ to get a system $\mathcal{S}^{\prime}=\left\{S_{1}^{\prime}, \ldots, S_{m}^{\prime}\right\}$ with the attractor $K^{\prime}$, such that the set $S_{i}^{\prime}\left(K^{\prime}\right) \cap S_{j}^{\prime}\left(K^{\prime}\right)$ is empty?

To find the answer to this question, we consider the system $\mathcal{S}=\mathcal{S}_{0}$ as an element of a parametrized family $\mathcal{S}_{t}=\left\{S_{1, t}, \ldots, S_{m, t}\right\}$, where the parameter $t$ assumes the values from some subset $D$ in $\mathbb{R}^{n}$. We denote the attractor of the system $\mathcal{S}_{t}$ by $K_{t}$. We search for the conditions under which $S_{i, t}\left(K_{t}\right) \cap S_{j, t}\left(K_{t}\right)$ is empty for almost all $t \in D$. In this case we say that $S_{i, t}\left(K_{t}\right)$ and $S_{j, t}\left(K_{t}\right)$ are disjoint in general position.

Particularly, this occurs when Hausdorff dimension of the set $\Delta=\left\{t \in D: S_{i, t}\left(K_{t}\right) \cap S_{j, t}\left(K_{t}\right) \neq \infty\right\}$ is less than $\operatorname{dim}_{H}(D)$.

It is possible to make an estimate of $\operatorname{dim}_{H}(\Delta)$ in terms of upper bound for similarity dimensions of the systems $\left\{\mathcal{S}_{t}: t \in D\right\}$. The method for finding such estimates is based on General Position Theorem [7, which was initially introduced in [11].

\section{Definitions and notations}

Let $(X, d)$ be a complete metric space. A mapping $S: X \rightarrow X$ is a contraction if $\operatorname{Lip} S<1$ and it is called a similarity if $d(S(x), S(y))=r d(x, y)$ for all $x, y \in X$ and some fixed $r$.

Let $\mathcal{S}=\left\{S_{1}, \ldots, S_{m}\right\}$ be a system of contractions in a complete metric space $(X, d)$. A nonempty compact set $K \subset X$ is called the attractor of the system $\mathcal{S}$, if $K=\bigcup_{i=1}^{m} S_{i}(K)$. By Hutchinson's Theorem [6], the attractor $K$ is uniquely defined by the system $\mathcal{S}$. We also call the set $K$ self-similar with respect to $\mathcal{S}$, when all $S_{i}$ are similarities.

Multiindices. Given a system $\mathcal{S}=\left\{S_{1}, \ldots, S_{m}\right\}, I=\{1, \ldots, m\}$ is the set of indices, $I^{*}=\bigcup_{n=1}^{\infty} I^{n}$ is the set of all finite $I$-tuples, or multiindices $\mathbf{j}=j_{1} j_{2} \ldots j_{n}$. By $\mathbf{i j}$ we denote the concatenation of the corresponding multiindices; we write $\mathbf{i} \sqsubset \mathbf{j}$, if $\mathbf{j}=\mathbf{i k}$ for some $\mathbf{k} \in I^{*}$; we say that $\mathbf{i}$ and $\mathbf{j}$ are incomparable, if neither $\mathbf{i} \sqsubset \mathbf{j}$ nor $\mathbf{j} \sqsubset \mathbf{j}$; by $\mathbf{i} \wedge \mathbf{j}$ we mean the maximal $\mathbf{k}$ for which $\mathbf{k} \sqsubset \mathbf{i}$ and $\mathbf{k} \sqsubset \mathbf{j}$; by - $\mathbf{i}$ - we denote the length of $\mathbf{i}$.

We write $S_{\mathbf{j}}=S_{j_{1} j_{2} \ldots j_{n}}=S_{j_{1}} S_{j_{2}} \ldots S_{j_{n}}$ and for the set $A \subset X$ we denote $S_{\mathbf{j}}(A)$ by $A_{\mathbf{j}}$; given a set of $\mathrm{m}$ ratios $\left\{r_{k}, k \in I\right\}$ we write $r_{\mathbf{j}}=r_{j_{1}} r_{j_{2}} \ldots r_{j_{n}}$.

The Index Space. $I^{\infty}=\left\{\mathbf{i}=i_{1} i_{2} \ldots: \quad i_{k} \in I\right\}$ is the index space; $\pi: I^{\infty} \rightarrow K$ is the index map, which sends $\mathbf{i} \in I^{\infty}$ to the point $\bigcap_{n=1}^{\infty} K_{i_{1} \ldots i_{n}}$. For a given vector $\mathbf{r}=\left(r_{1}, \ldots, r_{m}\right) \in(0,1)^{m}$ we define a metrics $\rho_{\mathbf{r}}$ on $I^{\infty}$ by $\rho_{\mathbf{r}}(\boldsymbol{\alpha}, \boldsymbol{\beta})=r_{\boldsymbol{\alpha} \wedge \boldsymbol{\beta}}$. The set $I^{\infty}$ supplied with this metrics will be denoted by $I_{\rho_{\mathbf{r}}}^{\infty}$. Let $s_{\mathbf{r}}$ denote the unique solution of the Moran equation $r_{1}^{s}+\cdots+r_{m}^{s}=1$. Then, by [4, Theorem 6.4.3], $\operatorname{dim}_{H} I_{\rho_{\mathbf{r}}}^{\infty}=s_{\mathbf{r}}$.

${ }^{*}$ Supported by Russian Foundation of Basic Research projects 18-01-00420 and 18-501-51021 
Separation conditions. Denote $\mathcal{F}=\left\{S_{\mathbf{i}}^{-1} S_{\mathbf{j}}: \mathbf{i}, \mathbf{j} \in I^{*}\right\}$. Then the system $\mathcal{S}=\left\{S_{1}, \ldots, S_{m}\right\}$ of contraction similarities has the Weak Separation Property (WSP) iff Id $\notin \overline{\mathcal{F} \backslash \text { Id }}[12$. The system $\mathcal{S}$ satisfies Open Set Condition (OSC) if there is an open set $V$ such that for any $i \in I, S_{i}(V) \in V$ and for any non-equal $i, j \in I, S_{i} \cap S_{j}(V)=\varnothing$. The system satisfies Strong Separation Condition (SSC), if for any non-equal $i, j \in I, K_{i} \cap K_{j}=\varnothing$. There are well-known implications (SSC) $\rightarrow(\mathrm{OSC})$ and $(\mathrm{OSC}) \rightarrow(\mathrm{WSP})[1,9,12]$

\section{General position theorem}

We begin with a simple example. Let $A, B$ be compact subsets in $\mathbb{R}^{n}$, and the set $B$ is being translated by a vector $t \in D$, where $D \subset \mathbb{R}^{n}$. We wish to understand, how large can be the set of parameters $\Delta=\{t \in D: A \cap(B+t) \neq \varnothing\}$, which we will call the set of exceptional parameters.

It's easy to see that $A \cap(B+t) \neq \varnothing$ is equivalent to: " there are such $a \in A, b \in B$ that $a=b+t$ ". Finding $t$ from this equation, we see that $\Delta=\{a-b: a \in A, b \in B\}$. How to evaluate the Hausdorff dimension of the set $\Delta$ in terms of $A$ and $B$ ?

For that reason we introduce the map $f: A \times B \rightarrow \Delta, f(a, b)=a-b$. Since $f$ is Lipschitz, $\operatorname{dim}_{H} \Delta \leq \operatorname{dim}_{H}(A \times B)$, and if the product $A \times B$ has the dimension less than $\operatorname{dim}_{H} D$, then $A$ and $B+t$ are disjoint for almost all $t \in D$.

We will extend this approach to a very general situation, taking a normed linear space $\mathcal{M}$ instead of $\mathbb{R}^{n}$, replacing $A$ and $B$ by metric spaces $\left(L_{1}, \sigma_{1}\right),\left(L_{2}, \sigma_{2}\right)$ and finding the set $\Delta$ for parametrized families $A_{t}=\varphi_{1}\left(t, L_{1}\right)$ and $B_{t}=\varphi_{2}\left(t, L_{2}\right)$ instead of $A$ and $B+t$. [7]:

Theorem 1. Let the Cartesian products of metric spaces $(D, \rho),\left(L_{1}, \sigma_{1}\right),\left(L_{2}, \sigma_{2}\right)$ be supplied with the canonical metrisation (see [8, §21.VI, (1)]). Let continuous maps $\varphi_{1}: D \times L_{1} \rightarrow \mathcal{M}$ and $\varphi_{2}$ : $D \times L_{2} \rightarrow \mathcal{M}$ to the normed linear space $(\mathcal{M},\|\|$.$) be such that:$

(a) there are $C_{0}>0$ and $\alpha>0$ such that for any $i=1,2$ and for all $(\xi, x),(\xi, y)$ in $D \times L_{i}$ the estimate holds

$$
\left\|\varphi_{i}(\xi, x)-\varphi_{i}(\xi, y)\right\| \leq C_{0}\left[\sigma_{i}(x, y)\right]^{\alpha}
$$

(uniform $\alpha$-Hölder continuity condition);

(b) there are such $M_{0}>0$ and $\beta>0$ that for any $\left(x_{1}, x_{2}\right) \in L_{1} \times L_{2}$ and $\xi, \xi^{\prime} \in D$ the function

$$
\Phi\left(\xi, x_{1}, x_{2}\right):=\varphi_{1}\left(\xi, x_{1}\right)-\varphi_{2}\left(\xi, x_{2}\right)
$$

on the set $D \times L_{1} \times L_{2}$ satisfies the condition

$$
\left\|\Phi\left(\xi^{\prime}, x_{1}, x_{2}\right)-\Phi\left(\xi, x_{1}, x_{2}\right)\right\| \geq M_{0}\left[\rho\left(\xi^{\prime}, \xi\right)\right]^{\beta} .
$$

Then Hausdorff dimension of the set $\Delta:=\left\{\xi \in D: \varphi_{1}\left(\xi, L_{1}\right) \cap \varphi_{2}\left(\xi, L_{2}\right) \neq \varnothing\right\}$ satisfies

$$
\operatorname{dim}_{H} \Delta \leq \min \left\{(\beta / \alpha) \operatorname{dim}_{H}\left(L_{1} \times L_{2}\right), \operatorname{dim}_{H} D\right\} .
$$

Moreover, if the spaces $\left(L_{1}, \sigma_{1}\right),\left(L_{2}, \sigma_{2}\right)$ are compact, $\Delta$ is closed in $D$.

\section{Proof.}

Put $\tilde{\Delta}:=\left\{\left(\xi, x_{1}, x_{2}\right) \in D \times L_{1} \times L_{2}: \varphi_{1}\left(\xi, x_{1}\right)=\varphi_{2}\left(\xi, x_{2}\right)\right\}=\left\{\left(\xi, x_{1}, x_{2}\right) \in D \times L_{1} \times L_{2}: \Phi\left(\xi, x_{1}, x_{2}\right)=\right.$ $0\}$ and notice that $\Delta=\operatorname{pr}_{1} \tilde{\Delta}$, where $\operatorname{pr}_{1}: D \times L_{1} \times L_{2} \rightarrow D$ is the canonical projection.

Applying canonical projection $\operatorname{pr}_{2}: D \times\left(L_{1} \times L_{2}\right) \rightarrow L_{1} \times L_{2}$ we obtain a set $\Delta_{L}:=\operatorname{pr}_{2}(\tilde{\Delta})$, that is,

$$
\Delta_{L}=\left\{\left(x_{1}, x_{2}\right) \in L_{1} \times L_{2} \mid \exists \xi \in D: \varphi_{1}\left(\xi, x_{1}\right)=\varphi_{2}\left(\xi, x_{2}\right)\right\} .
$$

The maps $\pi_{D}=\left.\operatorname{pr}_{1}\right|_{\tilde{\Delta}}: \tilde{\Delta} \rightarrow \Delta$ and $\pi_{L}=\left.\operatorname{pr}_{2}\right|_{\tilde{\Delta}}: \tilde{\Delta} \rightarrow \Delta_{L}$ are continuous open maps (by properties of canonical projections). Let us show that $\pi_{L}$ is a bijection. Indeed, if for $\left(\xi^{\prime}, x_{1}^{\prime}, x_{2}^{\prime}\right) \in \tilde{\Delta}$ and $\left(\xi^{\prime \prime}, x_{1}^{\prime \prime}, x_{2}^{\prime \prime}\right) \in \tilde{\Delta}$ the equality $\pi_{L}\left(\xi^{\prime}, x_{1}^{\prime}, x_{2}^{\prime}\right)=\pi_{L}\left(\xi^{\prime \prime}, x_{1}^{\prime \prime}, x_{2}^{\prime \prime}\right)$ holds, then $\left(x_{1}^{\prime}, x_{2}^{\prime}\right)=\left(x_{1}^{\prime \prime}, x_{2}^{\prime \prime}\right)=$ $\left(x_{1}, x_{2}\right)$, whereas $\Phi\left(\xi^{\prime}, x_{1}, x_{2}\right)=0=\Phi\left(\xi^{\prime \prime}, x_{1}, x_{2}\right)$. Then from (11) it follows that $0=\| \Phi\left(\xi^{\prime}, x_{1}, x_{2}\right)-$ $\Phi\left(\xi^{\prime \prime}, x_{1}, x_{2}\right) \| \geq M_{0}\left[\rho\left(\xi^{\prime}, \xi^{\prime \prime}\right)\right]^{\beta}$, that is, $\rho\left(\xi^{\prime}, \xi^{\prime \prime}\right)=0$. This means that $\xi^{\prime}=\xi^{\prime \prime}$. 
Since every open bijective continuous map is a homeomorphism (see [8, $\S 13 . \mathrm{XIII}]$ ), the maps $\pi_{L}$ and $\pi_{L}^{-1}$ are homeomorphisms.

Now we find Hölder continuity estimate for a map $g=\pi_{D} \circ \pi_{L}^{-1}: \Delta_{L} \rightarrow \Delta$. Let $\xi^{\prime}=g\left(x_{1}^{\prime}, x_{2}^{\prime}\right)$ and $\xi=g\left(x_{1}, x_{2}\right)$. Then $\Phi\left(\xi^{\prime}, x_{1}^{\prime}, x_{2}^{\prime}\right)=0=\Phi\left(\xi, x_{1}, x_{2}\right)$ and, particularly, $\varphi_{1}\left(\xi^{\prime}, x_{1}^{\prime}\right)=\varphi_{2}\left(\xi^{\prime}, x_{2}^{\prime}\right)$. The inequality (1) gives an estimate

$$
\begin{gathered}
M_{0}\left[\rho\left(\xi^{\prime}, \xi\right)\right]^{\beta} \leq\left\|\Phi\left(\xi^{\prime}, x_{1}, x_{2}\right)-\Phi\left(\xi, x_{1}, x_{2}\right)\right\|=\left\|\Phi\left(\xi^{\prime}, x_{1}, x_{2}\right)-0\right\|= \\
=\left\|\varphi_{1}\left(\xi^{\prime}, x_{1}\right)-\varphi_{2}\left(\xi^{\prime}, x_{2}\right)\right\| \leq\left\|\varphi_{1}\left(\xi^{\prime}, x_{1}\right)-\varphi_{1}\left(\xi^{\prime}, x_{1}^{\prime}\right)\right\|+\left\|\varphi_{1}\left(\xi^{\prime}, x_{1}^{\prime}\right)-\varphi_{2}\left(\xi^{\prime}, x_{2}\right)\right\|= \\
=\left\|\varphi_{1}\left(\xi^{\prime}, x_{1}\right)-\varphi_{1}\left(\xi^{\prime}, x_{1}^{\prime}\right)\right\|+\left\|\varphi_{2}\left(\xi^{\prime}, x_{2}^{\prime}\right)-\varphi_{2}\left(\xi^{\prime}, x_{2}\right)\right\| .
\end{gathered}
$$

Applying the condition (a), we get the inequality

$$
M_{0}\left[\rho\left(\xi^{\prime}, \xi\right)\right]^{\beta} \leq C_{0}\left[\sigma_{1}\left(x_{1}, x_{1}^{\prime}\right)\right]^{\alpha}+C_{0}\left[\sigma_{2}\left(x_{2}, x_{2}^{\prime}\right)\right]^{\alpha} \leq 2 C_{0}\left[\sqrt{\sigma_{1}\left(x_{1}, x_{1}^{\prime}\right)^{2}+\sigma_{2}\left(x_{2}, x_{2}^{\prime}\right)^{2}}\right]^{\alpha} .
$$

Denoting by $\tilde{\sigma}$ the metrics of Cartesian product of the spaces $\left(L_{1}, \sigma_{1}\right)$ and $\left(L_{2}, \sigma_{2}\right)$, we get Hölder continuity estimate of the map $g$ :

$$
\rho\left(g\left(x_{1}^{\prime}, x_{2}^{\prime}\right), g\left(x_{1}, x_{2}\right)\right) \leq\left(2 C_{0} / M_{0}\right)^{1 / \beta}\left[\tilde{\sigma}\left(\left(x_{1}^{\prime}, x_{2}^{\prime}\right),\left(x_{1}, x_{2}\right)\right)\right]^{\alpha / \beta} .
$$

Applying [5, Proposition 2.3] and the inequality $\operatorname{dim}_{H} \Delta_{L} \leq \operatorname{dim}_{H}\left(L_{1} \times L_{2}\right)$, we get the desired relation (2):

$$
\operatorname{dim}_{H} \Delta=\operatorname{dim}_{H} g\left(\Delta_{L}\right) \leq(\beta / \alpha) \operatorname{dim}_{H}\left(L_{1} \times L_{2}\right) \text { and } \operatorname{dim}_{H} \Delta \leq \operatorname{dim}_{H} D .
$$

Since the maps $\varphi_{i}$ are continuous, $\Phi$ is continuous too. The set $\tilde{\Delta}$ is closed in $D \times L_{1} \times L_{2}$ as a set of zeros of $\Phi$. If $L_{1} \times L_{2}$ is compact, the map $\pi_{D}$ is proper [3, Corollary 8 §10], therefore the set $\Delta=\pi_{D} \tilde{\Delta}$ is closed in $D$ (by properties of canonical projections).

\section{Remarks.}

1. We see from the inequality (2) that if the product $L_{1} \times L_{2}$ has sufficiently small dimension, then the sets $\varphi\left(t, L_{1}\right)$ and $\psi\left(t, L_{2}\right)$ do not intersect for almost all $t \in D$. The proof of the inequality (2) in the Theorem does not use the condition that the functions $\varphi_{1}$ and $\varphi_{2}$ are continuous with respect to the metrization of product spaces, so this condition may be omitted. It is needed only to show that $\Delta$ is closed in $D$.

2. The condition (b) in the Theorem may be considered as a form of transversality condition [10, where $D \subset \mathbb{R}^{n}$ is an open set, $\beta=1$ and $\varphi_{i}(i=1,2)$ are the address maps to different copies of a self-similar set, depending of a parameter $\xi \in D$.

3. Notice that the only information required of the parameter space $D$ is its Hausdorff dimension. Moreover, if $\operatorname{dim}_{H} D=s$ but the measure $H^{s}(D)$ is zero, we take some $s^{\prime}$ satisfying $\operatorname{dim}_{H} \Delta<s^{\prime}<s$ to see that $\Delta$ is negligible in $D$ in a sense that $H^{s^{\prime}}(D)=\infty$ and $H^{s^{\prime}}(\Delta)=0$.

For more easy understanding of the main idea ot the Theorem 1 we apply it to much more simplified settings. Nevertheless even the following simplified form will be useful for many applications:

Corollary 2. Let $A, B, D$ be some subsets of $\mathbb{R}^{n}$. Let the map $\varphi: D \times B \rightarrow \mathbb{R}^{n}$ be such that:

(a) there is $C_{0}>0$ such that for any $x, y \in B$ and $t \in D,\|\varphi(t, x)-\varphi(t, y)\| \leq C_{0}\|x-y\|$

(b) there is such $M_{0}>0$ that for any $x \in B$ and $t, t^{\prime} \in D$

$$
\left\|\varphi\left(t^{\prime}, x\right)-\varphi(t, x)\right\| \geq M_{0}\left\|t^{\prime}-t\right\| .
$$

Then Hausdorff dimension of the set $\Delta:=\{t \in D: \varphi(t, B) \cap A \neq \varnothing\}$ satisfies

$$
\operatorname{dim}_{H} \Delta \leq \min \left\{\operatorname{dim}_{H}(A \times B), \operatorname{dim}_{H} D\right\}
$$

Moreover, if $A$ and $B$ are compact and the map $\varphi$ is continuous, then $\Delta$ is closed in $D$. 
One can consider several specific applications which may be derived from the Corollary 2.

Example 1. If $A, B \subset \mathbb{C}$ and $0 \notin \bar{A}$ and $\operatorname{dim}_{H} A \times B<2$ then for Lebesgue almost all $z \in \mathbb{C}$ : $B \cap z A=\varnothing$.

Indeed, let $M_{0}=\inf \{|z|: z \in A\}$ and for some $C_{0}>0$, let $D=\left\{z:|z|<C_{0}\right\}$. Then the conditions (a) and (b) of the Corollary 2 are fulfilled. Therefore, if $\operatorname{dim}_{H}(A \times B)<2$ then for Lebesgue almost all $z \in D$ the sets $A$ and $B$ are disjoint. Letting $C_{0}$ tend to infinity we get that the statement is true for Lebesgue almost all $z \in \mathbb{C}$.

Example 2. If $A, B \subset \mathbb{R}^{n}, M_{2}>M_{1}>0$, a map $f: B \times \mathbb{R}^{n} \rightarrow \mathbb{R}^{n}$ is $M_{1}$-Lipschitz, and $\operatorname{dim}_{H}(A \times B)<$ $n$, then the set $\Delta=\left\{t \in \mathbb{R}^{n}: M_{2} t+f(B, t) \cap A \neq \varnothing\right\}$ has zero measure in $\mathbb{R}^{n}$.

In this case the conditions (a),(b) are fulfilled with $C_{0}=M_{1}$ and $M_{0}=M_{2}-M_{1}$. Since the set $\Delta$ can be represented also as $\left\{t \in \mathbb{R}^{n}: f(B, t) \cap M_{2} t+A \neq \varnothing\right\}$ this means that if $A$ moves faster that the set $B$ is deformed, for almost all $t$ the set $A$ escapes the intersection with the set $f(B, t)$.

Example 3. Suppose $A, B \subset \mathbb{R}^{n}$, a map $F: \mathbb{R}^{n} \rightarrow \mathbb{R}^{n}$ is bi-Lipschitz, and $f: B \times \mathbb{R}^{m}$ is defined by $f(x, t)=F(x+t) \cdot \operatorname{dim}_{H}(A \times B)<n$, then the set $\Delta=\left\{t \in \mathbb{R}^{n}: f(B, t) \cap A \neq \varnothing\right\}$ has zero measure in $\mathbb{R}^{n}$.

In this case we can interpret $f(B, t)$ as a bi-Lipschitz distortion of a translation of the set $B$ by a vector $t$.

\section{Application of General Position Theorem to self-similar sets}

The General Position Theorem is a tool for treating more complicated cases, than those in which one of the sets undergoes simple rigid motions or similarities or translations in some curvilinear coordinates. It works with the attractors $K_{t}$ of parametrized systems $\mathcal{S}_{t}$ of contraction maps. These attractors need not be even homeomorphic to each other for different values of the parameter $t$.

To analyze transformations of the attractors of such systems, we define the following settings for parametrized families:

(S1). Let $\mathcal{S}_{t}=\left\{S_{1, t}, \ldots, S_{m, t}\right\}$ be a system of contraction maps in $\mathbb{R}^{n}$, depending on the parameter $t \in D \subset \mathbb{R}^{n}$ and let $K_{t}$ be its attractor.

(S2) Suppose there is a compact set $V$ such that for any $k \in I$ and any $t \in D, S_{k, t}(V) \subset V$.

(S3) There is a vector $\mathbf{r}=\left(r_{1}, \ldots, r_{m}\right)$ such that for any $t \in D$ and for any $k \in I$, Lip $S_{k_{t}} \leq r_{k}<1$. Let $\bar{r}=\max \left\{r_{1}, \ldots, r_{m}\right\}$.

(S4) There is such $C>0$ that for any $x \in V, k \in I$ and for any $t, t^{\prime} \in D,\left\|S_{k, t^{\prime}}(x)-S_{k, t}(x)\right\| \leq C\left\|t^{\prime}-t\right\|$

\subsection{Moving subpieces apart from each other.}

First notice that it follows from the settings $(\mathbf{S 1}),(\mathbf{S 3})$ that all the address maps are Lipschitz with a constant equal to $\operatorname{diam}(K)$ :

Lemma 3. If the settings (S1),(S3) are fulfilled then the map $\pi: I_{\rho_{\mathbf{r}}}^{\infty} \rightarrow K$ is diam(K)-Lipschitz.

Proof: (cf. [4, Ex. 4.2.4]). Suppose $\boldsymbol{\alpha} \wedge \boldsymbol{\beta}=\mathbf{j}$, so $\boldsymbol{\alpha}=\mathbf{j} \boldsymbol{\alpha}^{\prime}$ and $\boldsymbol{\beta}=\mathbf{j} \boldsymbol{\beta}^{\prime}$. From $\rho_{\mathbf{r}}\left(\boldsymbol{\alpha}^{\prime}, \boldsymbol{\beta}^{\prime}\right)=1$ we get $\|\pi(\boldsymbol{\alpha})-\pi(\boldsymbol{\beta})\|=\| S_{\mathbf{j}}\left(\pi\left(\boldsymbol{\alpha}^{\prime}\right)\right)-S_{\mathbf{j}}\left(\pi\left(\boldsymbol{\beta}^{\prime}\right)\right) \mid \leq r_{\mathbf{j}} \operatorname{diam}(K)=\operatorname{diam}(K) \rho_{\mathbf{r}}(\boldsymbol{\alpha}, \boldsymbol{\beta})$.

To evaluate the distance between the points in $K_{t}$ and $K_{t^{\prime}}$ having the same addresses, we use the Displacement Theorem for parametrized families (cf. [7, Theorem 17]):

Theorem 4. Suppose the settings (S1)-(S4) hold. Then for any $\boldsymbol{\alpha} \in I^{\infty}$ and any $t, t^{\prime} \in D$ we have

$$
\left\|\pi_{t^{\prime}}(\boldsymbol{\alpha})-\pi_{t}(\boldsymbol{\alpha})\right\| \leq \frac{C\left\|t^{\prime}-t\right\|}{1-\bar{r}} .
$$


Proof: Take $\boldsymbol{\alpha}=i_{1} i_{2} \ldots$ and denote $\boldsymbol{\alpha}_{k}=i_{k} i_{k+1} \ldots$ Since $\pi_{t}\left(\boldsymbol{\alpha}_{k}\right)=S_{i_{k}}^{t} \pi_{t}\left(\boldsymbol{\alpha}_{k+1}\right),\left\|\pi_{t}\left(\boldsymbol{\alpha}_{k}\right)-\pi_{t^{\prime}}\left(\boldsymbol{\alpha}_{k}\right)\right\| \leq\left\|S_{i_{k}}^{t} \pi_{t}\left(\boldsymbol{\alpha}_{k+1}\right)-S_{i_{k}}^{t} \pi_{t^{\prime}}\left(\boldsymbol{\alpha}_{k+1}\right)\right\|+\| S_{i_{k}}^{t} \pi_{t^{\prime}}\left(\boldsymbol{\alpha}_{k+1}\right)-$ $S_{i_{k}}^{t^{\prime}} \pi_{t^{\prime}}\left(\boldsymbol{\alpha}_{k+1}\right) \|$, so $\left\|\pi_{t}\left(\boldsymbol{\alpha}_{k}\right)-\pi_{t^{\prime}}\left(\boldsymbol{\alpha}_{k}\right)\right\| \leq r_{i_{k}}\left\|\pi_{t}\left(\boldsymbol{\alpha}_{k+1}\right)-\pi_{t^{\prime}}\left(\boldsymbol{\alpha}_{k+1}\right)\right\|+C\left\|t^{\prime}-t\right\|$ for any $k \in \mathbb{N}$.

Therefore $\left\|\pi_{t}(\boldsymbol{\alpha})-\pi_{t^{\prime}}(\boldsymbol{\alpha})\right\| \leq \bar{r}^{n+1}\left\|\pi_{t}\left(\boldsymbol{\alpha}_{n+1}\right)-\pi_{t^{\prime}}\left(\boldsymbol{\alpha}_{n+1}\right)\right\|+C\left\|t^{\prime}-t\right\| \sum_{k=0}^{n} \bar{r}^{k}$, which becomes (5) as $k$ tends to $\infty$.

The following Theorem gives the conditions under which the pieces $K_{\mathbf{j}, t}$ and $K_{\mathbf{k}, t}$ are disjoint for almost all $t \in D$ :

Theorem 5. Suppose the settings (S1)-(S4) hold. Let $\mathbf{j}, \mathbf{k} \in I^{*}$ be incomparable multiindices. Suppose there are such $c_{\mathbf{j}}>0, C_{\mathbf{k}}>0$ that for any $x \in V$ and for any $t, t^{\prime} \in D$,

$$
\left\|S_{\mathbf{k}}^{t^{\prime}}(x)-S_{\mathbf{k}}^{t}(x)\right\| \leq C_{\mathbf{k}}\left\|t^{\prime}-t\right\| \text { and }\left\|S_{\mathbf{j}, t^{\prime}}(x)-S_{\mathbf{j}, t}(x)\right\| \geq c_{\mathbf{j}}\left\|t^{\prime}-t\right\|
$$

If

$$
c_{\mathbf{j}}-C_{\mathbf{k}}-\frac{\left(r_{\mathbf{j}}+r_{\mathbf{k}}\right) C}{1-\bar{r}}>0
$$

and $s_{\mathbf{r}}<\operatorname{dim}_{H}(D) / 2$, then $K_{\mathbf{j}} \cap K_{\mathbf{k}}=\varnothing$ for almost all $t \in D$.

Proof: $\quad$ Let $\varphi(t, x)=S_{\mathbf{k}, t}\left(\pi_{t}(x)\right), \quad \psi(t, x)=S_{\mathbf{j}, t}\left(\pi_{t}(x)\right), \quad \Phi(t, x, y)=\varphi(t, x)-\psi(t, y)$, $\Delta=\left\{t \in D: K_{\mathbf{j}} \cap K_{\mathbf{k}} \neq \varnothing\right\}$. Note that

$$
\begin{aligned}
\left\|\Phi\left(t^{\prime}, x, y\right)-\Phi(t, x, y)\right\| & \geq\left\|\psi\left(t^{\prime}, y\right)-\psi(t, y)\right\|-\left\|\varphi\left(t^{\prime}, x\right)-\varphi(t, x)\right\| \\
\left\|\varphi\left(t^{\prime}, x\right)-\varphi(t, x)\right\| & \leq\left\|S_{\mathbf{k}, t^{\prime}}\left(\pi_{t^{\prime}}(x)\right)-S_{\mathbf{k}, t}\left(\pi_{t^{\prime}}(x)\right)\right\|+\left\|S_{\mathbf{k}, t}\left(\pi_{t^{\prime}}(x)\right)-S_{\mathbf{k}, t}\left(\pi_{t}(x)\right)\right\| ; \\
\left\|\psi\left(t^{\prime}, x\right)-\psi(t, x)\right\| & \geq\left\|S_{\mathbf{j}, t^{\prime}}\left(\pi_{t^{\prime}}(x)\right)-S_{\mathbf{j}, t}\left(\pi_{t^{\prime}}(x)\right)\right\|-\left\|S_{\mathbf{j}, t}\left(\pi_{t^{\prime}}(x)\right)-S_{\mathbf{j}, t}\left(\pi_{t}(x)\right)\right\| .
\end{aligned}
$$

From Theorem 4 we have upper estimates

$$
\left\|S_{\mathbf{k}, t}\left(\pi_{t^{\prime}}(x)\right)-S_{\mathbf{k}, t}\left(\pi_{t}(x)\right)\right\| \leq \frac{r_{\mathbf{k}} C\left\|t^{\prime}-t\right\|}{1-\bar{r}} \quad \text { and } \quad\left\|S_{\mathbf{j}, t}\left(\pi_{t^{\prime}}(x)\right)-S_{\mathbf{j}, t}\left(\pi_{t}(x)\right)\right\| \leq \frac{r_{\mathbf{j}} C\left\|t^{\prime}-t\right\|}{1-\bar{r}}
$$

Combining them with inequalities ([6), we obtain

$$
\left\|\Phi\left(t^{\prime}, x, y\right)-\Phi(t, x, y)\right\| \geq\left(c_{\mathbf{j}}-C_{\mathbf{k}}-\frac{C\left(r_{\mathbf{k}}+r_{\mathbf{j}}\right)}{1-\bar{r}}\right)\left\|t^{\prime}-t\right\|
$$

Applying the Theorem 1 with $\alpha=\beta=1$ we get $\operatorname{dim}_{H} \Delta<2 \operatorname{dim}_{H}\left(I_{\rho_{\mathbf{r}}}^{\infty}\right)=2 s_{\mathbf{r}}$.

Since $s_{\mathbf{r}}<\operatorname{dim}_{H}(D) / 2$ we get $H^{2 s_{\mathbf{r}}}(\Delta)=0$ and at the same time $H^{2 s_{\mathbf{r}}}(D)=\infty$.

\subsubsection{The case when the parameters are translation vectors.}

We consider the case is when the initial system $\mathcal{S}=\left\{S_{1}, \ldots, S_{m}\right\}$ consists of the contraction maps $S_{k}$ in $\mathbb{R}^{n}$; and we consider a parametrized system $\mathcal{S}_{t}=\left\{S_{1, t}, \ldots, S_{m, t}\right\}$ where each $S_{k, t}$ is defined by the formula $S_{k, t}(x)=S_{k}(x)+t_{k}$, where $\boldsymbol{t}=\left(t_{1}, \ldots, t_{m}\right) \in\left(\mathbb{R}^{n}\right)^{m}$. Translations have no effect upon the contraction ratios, therefore Lip $S_{k, t}=r_{k}$ for any $t$.

First we allow only one map, say $S_{m, t}$, to depend on the parameter $t$, leaving all others unchanged.

Corollary 6. Let $\mathcal{S}_{t}=\left\{S_{1}, \ldots, S_{m-1}, S_{m, t}(x)=S_{m}(x)+t\right\}$ be a system of contraction maps in $\mathbb{R}^{n}$, depending on the parameter $t \in \mathbb{R}^{n}$ and let $K_{t}$ be its attractor. Let $1 \leq k<m$. If $r_{k}+r_{m}+\bar{r}<1$ and $s_{\mathbf{r}}<n / 2$, then $K_{k, t} \cap K_{m, t}=\varnothing$ for almost all $t \in \mathbb{R}^{n}$.

Proof: For any open bounded $D \subset \mathbb{R}^{n}$ there is such $V \subset \mathbb{R}^{n}$ that the system $\mathcal{S}^{t}$ satisfies the settings (S1)-(S4); since $C=1$ the condition 7 of the Theorem 5 becomes equivalent to $r_{k}+r_{m}+\bar{r}<1$. Therefore $K_{k, t} \cap K_{m, t}=\varnothing$ for almost all $t \in D \subset \mathbb{R}^{n}$. The result does not depend on the choice of $D \subset \mathbb{R}^{n}$, so it holds for the whole $\mathbb{R}^{n}$.

Now, if we apply a translation by some vector $t_{k} \in \mathbb{R}^{n}$ to each map $S_{k} \in \mathcal{S}$, we obtain the following: 
Corollary 7. Let $\mathcal{S}=\left\{S_{1}, \ldots, S_{m-1}, S_{m}\right\}$ be a system of contraction maps in $\mathbb{R}^{n}$. Let $\boldsymbol{t}=\left\{t_{1}, \ldots, t_{m}\right\}$, where $t_{k} \in \mathbb{R}^{n}$. Let $S_{k, \boldsymbol{t}}(x)=S_{k}(x)+t_{k}$. Let $K_{\boldsymbol{t}}$ be the attractor of the system $\mathcal{S}_{\boldsymbol{t}}=\left\{S_{1, \boldsymbol{t}}, \ldots, S_{m, \boldsymbol{t}}\right\}$. If for any non-equal $j, k \in I, r_{j}+r_{k}+\bar{r}<1$ and $s_{\mathbf{r}}<n / 2$, then for almost all $\boldsymbol{t} \in \mathbb{R}^{m n}$, the system $\mathcal{S}$ satisfies Strong Separation Condition.

Proof: Notice that by Theorem 4 the maps $\pi_{j, t}: I^{\infty} \times \mathbb{R}^{n m} \rightarrow \mathbb{R}^{n}$ are continuous with respect to $\boldsymbol{t}$. Therefore the function $\rho_{j k}(\boldsymbol{t})=\min \left\{\left\|\pi_{j, \boldsymbol{t}}(\boldsymbol{\alpha})-\pi_{k, \boldsymbol{t}}(\boldsymbol{\beta})\right\|, \boldsymbol{\alpha}, \boldsymbol{\beta} \in I^{\infty}\right\}$ is continuous with respect to $\boldsymbol{t}$. Therefore the set $\Delta_{j k}=\rho^{-1}(\{0\})$ is closed in $\mathbb{R}^{n m}$. Since all of its $k$-slices $\left\{\left(t_{1}, . ., t_{k-1}, t, t_{k+1}, \ldots, t_{m}\right) \in\right.$ $\left.\Delta_{j k} ; t \in \mathbb{R}^{n}\right\}$ have zero Lebesgue $\mathrm{n}$-dimensional measure, the set $\Delta_{j k}$ has zero measure in $\mathbb{R}^{m n}$. Thus, the set $\Delta=\bigcup_{j, k \in I} \Delta_{j k}$ also has zero measure in $\mathbb{R}^{m n}$. Therefore, for almost all $\boldsymbol{t} \in \mathbb{R}^{m n}$, the system $\mathcal{S}_{\boldsymbol{t}}$ satisfies Strong Separation Condition.

\subsection{Non-empty overlaps of prescribed type.}

If we we get rid of all overlaps in a self-similar set, we obtain a system $\mathcal{S}$, which satisfy Strong Separation Condition and whose attractor $K$ is just a Cantor set. There is a mush more interesting case, when we use our techniques to obtain a system $\mathcal{S}$ of contraction maps which has the attractor $K$ such that the intersections of its pieces $K_{j}$ strictly follow some predefined pattern. The attractors of such systems possess a set of interesting properties and often they do not satisfy WSP. In this subsection we will see

a) how to find systems $\mathcal{S}$ for which two maps $S_{1}$ and $S_{2}$ commute and for which $S_{1}(K) \cap S_{2}(K)$ is exactly equal to $S_{12}(K)$ and

b) how to find systems $\mathcal{S}$ which do not satisfy OSC though all the pieces $S_{i}(K)$ are disjoint except $S_{1}(K) \cap S_{2}(K)$ which is a single point.

\subsubsection{Exact overlaps: an example}

First we consider the systems $\mathcal{S}$ in which two maps $S_{1}, S_{2}$ have a common fixed point and commute. (cf.[2]) Let the system $\mathcal{S}_{t}$ in $[0,1]$ consist of 3 maps: $S_{1}(x)=t x, S_{2}(x)=b x, S_{3}(x)=\frac{x+8}{9}$ in $\mathbb{R}$, where $b, t \in(0,1 / 9)$. It depends on the parameter $t$, while $b$ is a fixed value.

Since the maps $S_{1, t}$ and $S_{2}$ commute, we have the following inclusion:

$$
S_{1, t} S_{2}\left(K_{t}\right) \subseteq S_{1, t}\left(K_{t}\right) \cap S_{2}\left(K_{t}\right)
$$

We want to study for which $t \in(0,1 / 9)$ the inclusion (9) becomes equality. In this case we say the system $\mathcal{S}_{t}$ has exact overlap $S_{1}(K) \cap S_{2}(K)=S_{12}(K)$.

Notice that the same way as in ([7, Proposition 2(v)]),

$$
K_{t} \backslash\{0\}=\bigcup_{m, n=0}^{\infty} S_{1}^{m} S_{2}^{n}\left(K_{3, t}\right)
$$

Since $t, b<1 / 9$ and $K_{3} \subset[8 / 9,1]$, for any $m \neq n, S_{i}^{m}\left(K_{3}\right) \cap S_{i}^{n}\left(K_{3}\right)=\varnothing$ for $i=1,2$.

Following the argument of [7, Proposition 3] we obtain

Proposition 8. For the system $\mathcal{S}_{t}$ the following statements are equivalent:

(i) For any $m, n \in \mathbb{N}, S_{1}^{m}\left(K_{3}\right) \cap S_{2}^{n}\left(K_{3}\right)=\varnothing$;

(ii) $K=\{0\} \cup \bigsqcup_{m, n=0}^{\infty} S_{1}^{m} S_{2}^{n}\left(K_{3}\right)$;

(iii) For any $m, n \in \mathbb{N}, S_{1}^{m}(K) \cap S_{2}^{n}(K)=S_{1}^{m} S_{2}^{n}(K)$

Proposition 9. The system $\mathcal{S}_{t}$ has exact overlap $S_{1}(K) \cap S_{2}(K)=S_{12}(K)$ for Lebesgue almost all $t \in(0,1 / 9)$.

Proof: By Proposition 8 it suffices to find the set of those $t$, for which $S_{1}^{m}\left(K_{3}\right) \cap S_{2}^{n}\left(K_{3}\right)=\varnothing$ for any $m \neq n$. 
Take non-equal $m, n \in \mathbb{N}$ and let $D_{m n}=\left\{t \in(0,1 / 9): S_{1, t}^{m}([8 / 9,1]) \cap S_{2}^{n}([8 / 9,1]) \neq \varnothing\right\}$.

If $t \in D_{m n}$ then $\frac{8 b^{n}}{9} \leq t^{m} \leq \min \left\{\frac{9 b^{n}}{8}, \frac{1}{9^{m}}\right\}$. Put $\bar{t}=\left(\min \left\{\frac{9 b^{n}}{8}, \frac{1}{9^{m}}\right\}\right)^{1 / m}$.

To apply the Theorem 5 we interpret the case under consideration in terms of its settings: The system $\mathcal{S}_{t}$ depends on the parameter $t \in D_{m n}$.

The set $V=[0,1]$, the constant $C=1$. Since the vector $\mathbf{r}=(\bar{t}, b, 1 / 9)$, we have $s_{\mathbf{r}}<1 / 2$.

Further, $S_{\mathbf{j}}=S_{1, t}^{m}, S_{\mathbf{k}}=S_{2}^{n}$, therefore $r_{\mathbf{j}}=\bar{t}^{m}<\frac{9 b^{n}}{8}, r_{\mathbf{k}}=b^{n}$.

By definition, $c_{\mathbf{j}}=\inf _{t, t^{\prime} \in D_{m n}} \frac{t^{\prime m}-t^{m}}{t^{\prime}-t}=\inf _{t \in D_{m n}} m t^{m-1} \geq \inf _{t \in D_{m n}} \frac{t^{m}}{t}$.

Replacing $t^{m}$ by $\frac{8 b^{n}}{9}$ and $t$ in denominator by $1 / 9$, we get $c_{\mathbf{j}}>8 b^{n}$.

Since $C_{\mathbf{k}}=0$, we have $c_{\mathbf{j}}-C_{\mathbf{k}}-\frac{r_{\mathbf{j}}+r_{\mathbf{k}}}{1-\bar{r}}>\left(8-\frac{9 / 8+1}{8 / 9}\right) b^{n}$.

Therefore by Theorem 5 , the set $\Delta_{m n}=\left\{t \in D: S_{1, t}^{m}\left(K_{3, t}\right) \cap S_{2}^{n}\left(K_{3, t}\right) \neq \varnothing\right\}$ is a closed subset of $D_{m n}$ and $\operatorname{dim}_{H}\left(\Delta_{m n}\right)<1$.

Let $\Delta$ be the union of all $\Delta_{m n}$, where $m, n \in \mathbb{N}$ and $m \neq n$.

Then $\operatorname{dim}_{H}(\Delta) \leq 2 s_{\mathbf{r}}<1$ which implies the statement of the Proposition.

For almost all $t$ the systems $\mathcal{S}_{t}$ possess several remarkable properties:

1. Violation of WSP. Consider the set $D^{*}$ of those values of the parameter $t \in D \backslash \Delta$ for which $\frac{\log t}{\log b}$ is irrational. The set $D^{*}$ has full measure in $D$. For each $t \in D^{*}$ there are sequences of positive integers $l_{k}, n_{k}$ such that the sequence $t^{l_{k}} b^{-n_{k}}$ converges to 1 . Therefore the system $\mathcal{S}_{t}$ does not satisfy Weak Separation Property.

2. Measure and dimension. The Hausdorff dimension $s$ of the attractor $K_{t}, t \in D^{*}$ is equal to the solution of the equation $t^{x}+b^{x}-t^{x} b^{x}+9^{-x}=1$. Since the Weak Separation Property is violated, the Hausdorff measure $H^{s}\left(K_{t_{0}}\right)=0$.

3. All $K_{t}$ are isomorphic. For any two sets $K_{t_{1}}, K_{t_{2}}, t_{i} \in D^{*}$ there is a homeomorphism $\varphi: K_{t_{1}} \rightarrow K_{t_{2}}$, which agrees with the systems $\mathcal{S}_{1}$ and $\mathcal{S}_{2}$, i.e. for any $k=1, \ldots, 4$ and for any $x \in K_{t}$, $\varphi\left(S_{k, t}(x)=S_{k, t^{\prime}}(\varphi(x))\right.$.

We refer the reader to [7] for detailed proofs of the properties of such type of self-similar sets.

\subsubsection{One-point intersections: an example}

Take $p, q, r$ in $(0,1 / 36)$ and put $h=\frac{1}{2}, a=\frac{1}{3}$. Consider a system $\mathcal{S}=\left\{S_{1}, S_{2}, \ldots, S_{6}\right\}$ of contractions in $[0,1]$ whose equations are

$$
\begin{gathered}
S_{1}(x)=p x, \quad S_{2}(x)=a+r x, \quad S_{3}(x)=h-q x, \quad S_{4}(x)=h-r+r x, \\
S_{5}(x)=1-a-r x, \quad S_{6}(x)=1-r+r x
\end{gathered}
$$

The similarity dimension for any such system is strictly less than $1 / 2$.

Let $K$ be the attractor of the system $\mathcal{S}$ and $K_{i}=S_{i}(K)$ be its pieces. By the construction, $\{0,1\} \subset K \subset[0,1]$ and the pieces $K_{i}, i \in\{1,2,3,5,6\}$ are contained in disjoint segments of length $1 / 36$, while $K_{3} \cup K_{4} \subset[h-1 / 36, h]$ and $K_{3} \cap K_{4} \ni\{h\}$ which is the only possible non-empty intersection of the pieces.

We wish to know the set of those $p, q, r$ for which $K_{3} \cap K_{4}=\{h\}$. In this case we say that the system $\mathcal{S}$ has unique one-point intersection. 
If $\frac{\log p}{\log r} \notin \mathbb{Q}$, then the system $\mathcal{S}$ does not have WSP for any $q$. Indeed, consider the maps $H_{m}(x)=$ $S_{3} S_{1}^{m} S_{5}(x)$ and $G_{n}(x)=S_{4} S_{6}^{n} S_{2}(x)$. Notice that for any $q>0$ there is a sequence $\left(m_{k}, n_{k}\right) \in \mathbb{N}^{2}$, such that $p^{-m_{k}} r^{n_{k}+1}$ converges to $q$ as $k \rightarrow \infty$. Easy computation shows that if we choose such a sequence $\left(m_{k}, n_{k}\right)$, then the sequence

$$
G_{n_{k}}^{-1} H_{m_{k}}(x)=\frac{\left(r^{n_{k}+1}-p^{m_{k}} q\right)(1-a)}{r^{n_{k}+2}}+\frac{p^{m_{k}} q}{r^{n_{k}+1}} x
$$

converges to identity, which means violation of WSP.

Therefore we fix some $p, r \in(0,1 / 36)$ such that $\log _{r} p$ is irrational and consider a 1-parameter family of systems $\mathcal{S}_{q}, q \in(0,1 / 36)$, for which we show that for Lebesgue almost all $q \in(0,1 / 36)$ the system $\mathcal{S}_{q}$ has unique one-point intersection and does not have Weak Separation Property.

For the simplicity of notation, we denote the system under consideration by $\mathcal{S}$, keeping in mind that it depends on the parameter $q$ whenever it does not cause any ambiguity.

From the representation of the pieces $K_{3}$ and $K_{4}$ as unions of infinite sequences

$$
K_{3}=\{h\} \cup \bigcup_{m=0}^{\infty} S_{3} S_{1}^{m}\left(K \backslash K_{1}\right), \quad K_{4}=\{h\} \cup \bigcup_{n=0}^{\infty} S_{4} S_{6}^{n}\left(K \backslash K_{6}\right)
$$

we see that $K_{3} \cap K_{4}=\{h\}$ iff

for any $m, n \in \mathbb{N} \cup\{0\}$ and any $i \in I \backslash\{6\}, \quad j \in I \backslash\{1\}, \quad S_{3} S_{1}^{m}\left(K_{j}\right) \cap S_{4} S_{6}^{n}\left(K_{i}\right)=\varnothing$

Note that if $p^{m}[a q, q] \cap r^{n+1}[a, 1]=\varnothing$ then for any $i \in I \backslash\{6\}, j \in I \backslash\{1\}$ the intersections $S_{3} S_{1}^{m} S_{j}(K) \cap$ $S_{4} S_{6}^{n} S_{i}(K)$ are empty. Therefore, in search of those $q$ for which $S_{3} S_{1}^{m} S_{j}(K)$ and $S_{4} S_{6}^{n} S_{i}(K)$ may intersect, we can restrict the values of $q$ to the intervals

$$
D_{m n}(p, r):=\left(\frac{a r^{n+1}}{p^{m}}, \min \left(\frac{r^{n+1}}{a p^{m}}, 1 / 36\right)\right)
$$

We apply the Theorem 5 to the family $\mathcal{S}_{q}$ with the parameter set $D_{m n}(p, r)$ and to $S_{\mathbf{j}}=S_{3} S_{1}^{m}$ and $S_{\mathbf{k}}=S_{4} S_{6}^{n}$. We take $\mathbf{r}=(p, r, 1 / 36, r, r, r)$, therefore $s_{\mathbf{r}}<1 / 2$ and $\bar{r}=1 / 36$. We have $C=1, C_{\mathbf{k}}=0$ and $r_{\mathbf{k}}=r^{n+1}$. Now since the set $K_{j}$ lies in the interval $[a, 1]$, for $x \in K_{j}$ and $q^{\prime}, q \in D_{m n}(p, r)$ we have $\left|S_{\mathbf{j}, q^{\prime}}(x)-S_{\mathbf{j}, q}(x)\right|=\left|q^{\prime}-q\right| p^{m} x \geq\left|q^{\prime}-q\right| p^{m} a$, so $c_{\mathbf{j}}=p^{m} / 3$. Notice also that $r^{n+1}<3 p^{m} q$. Therefore

$$
c_{\mathbf{j}}-C_{\mathbf{k}}-\frac{r_{\mathbf{j}}+r_{\mathbf{k}}}{1-\bar{r}}>p^{m}\left(\frac{1}{3}-\frac{1}{35}-\frac{3}{35}\right)>\frac{p^{m}}{4}
$$

Therefore the set $\Delta_{m n}(p, r)=\left\{q: S_{3} S_{1}^{m}\left(K \backslash K_{1}\right) \cap S_{4} S_{6}^{n}\left(K \backslash K_{6}\right)\right.$ has the dimension less than $2 s_{\mathbf{r}}$. The same is true for the set $\Delta(p, r)$ which is a countable union of the sets $\Delta_{m n}(p, r)$.

This shows that

if $p, r \in(0,1 / 36)$ and $\frac{\log p}{\log r}$ is irrational then for Lebesgue almost all $q \in(0,1 / 36)$ the system $\mathcal{S}$ has totally disconnected attractor with unique one-point intersection and at the same time it does not satisfy weak separation property.

The reader may see that the properties similar to The properties $\mathbf{1}$. 2. $\mathbf{3}$. in the previous subsection are also valid for the systems, described above.

\section{References}

[1] Ch. Bandt, S. Graf, Self-similar sets 7. A characterization of self-similar fractals with positive Hausdorff measure, Proc. Amer. Math. Soc. 114:4 (1992), 995-1001. MR1100644

[2] B. Barany, Iterated function systems with non-distinct fixed points // J. Appl. Math. Anal. Appl. 383:1 (2011), pp. 244-258. 
[3] N. Bourbaki, General Topology: Chapters 14 // Springer-Verlag Berlin Heidelberg, 1987.

[4] G. Edgar, Measure, Topology, and Fractal Geometry // 2 ed, Springer-Verlag, New York, 2008, $272 \mathrm{p}$.

[5] K. J. Falconer, Fractal geometry: mathematical foundations and applications // J. Wiley and Sons, New York, 1990.

[6] J. Hutchinson, Fractals and self-similarity // Indiana Univ. Math. J. 30:5 (1981), pp. 713-747.

[7] K. G. Kamalutdinov, A. V. Tetenov, Twofold Cantor sets in $\mathbb{R}$, Siberian Electronic Mathematical Reports 15 (2018), pp. 801-814.

[8] K. Kuratowski, Topology, vol. 1, PWN and Acad. Press, (1966). MR0217751

[9] K. S. Lau and S. M. Ngai, Multifractal measures and a weak separation condition, Adv. Math. 141 (1999), 45-96. MR1667146

[10] K. Simon, B. Solomyak, M. Urbański, Hausdorff dimension of limit sets for parabolic IFS with overlaps, Pacific J. Math. 201:2 (2001), pp. 441-478.

[11] A. Tetenov, K. Kamalutdinov, D. Vaulin, Self-similar Jordan arcs which do not satisfy OSC, arXiv:1512.00290 (2015).

[12] M. P. W. Zerner, Weak separation properties for self-similar sets // Proc. Amer. Math. Soc. 124:11 (1996), pp. 3529-3539. 